\title{
Continuously tunable band gap in GaN/AIN (0001) superlattices via built-in electric field
}

\author{
X. Y. Cui, ${ }^{1}$ D. J. Carter,,${ }^{1,2}$ M. Fuchs, ${ }^{3}$ B. Delley, ${ }^{4}$ S. H. Wei, ${ }^{5}$ A. J. Freeman, ${ }^{6}$ and C. Stampfl ${ }^{1}$ \\ ${ }^{1}$ School of Physics, The University of Sydney, Sydney, New South Wales 2006, Australia \\ ${ }^{2}$ Nanochemistry Research Institute, Curtin University of Technology, Perth, Western Australia 6845, Australia \\ ${ }^{3}$ Fritz-Haber-Institut der Max-Planck-Gesellschaft, Faradayweg 4-6, 14195 Berlin, Germany \\ ${ }^{4}$ Paul Scherrer Institut, WHGA/123, CH-5232 Villigen PSI, Switzerland \\ ${ }^{5}$ National Renewable Energy Laboratory, 1617 Cole Boulevard, Golden, Colorado 80401, USA \\ ${ }^{6}$ Department of Physics and Astronomy, Northwestern University, Evanston, Illinois 60208-3112, USA \\ (Received 24 September 2009; revised manuscript received 9 March 2010; published 1 April 2010)
}

\begin{abstract}
Based on all-electron density-functional theory calculations using the generalized gradient approximation, we demonstrate the continuous tunability of the band gap and strength of the built-in electric field in GaN/AIN (0001) superlattices by control of the thickness of both the well ( $\mathrm{GaN})$ and barrier (AlN) regions. The effects of strain for these quantities are also studied. Calculations taking into account the self-interaction correction exhibit the same dependence on thickness. The calculated electric field strength values are in good agreement with recent experiments. Spontaneous polarization dominates the contribution to the electric field and the strain-induced piezoelectric polarization is estimated to contribute only about 5-10\%.
\end{abstract}

DOI: 10.1103/PhysRevB.81.155301

PACS number(s): 68.65.Cd, 71.15.Mb, 71.20.-b

\section{INTRODUCTION}

The polarization properties of (0001)-grown GaN-based heterostructures have been an intense research subject due to their crucial influence on, and thus affording a tailoring of, the electronic and optical performance of technologically important nitride devices. ${ }^{1-8}$ Macroscopic polarization, both of an intrinsic and piezoelectric nature, originates from polarization discontinuities at heterointerfaces, and manifests itself as built-in electrostatic fields across the heterostructure. The electric field, acting via the quantum-confined Stark effect (QCSE), ${ }^{9}$ changes the energies (spectra shift) and intensity (oscillator strength) (Ref. 10) of the emitted light. It can be engineered, for example, to influence favorably transistor characteristics $^{11}$ and spin injection. ${ }^{12}$

The intensity of the built-in electric fields and the effect on the resulting properties depend on the composition, structural geometry, and strain of the samples. Compared with using $(\mathrm{Ga}, \mathrm{Al}) \mathrm{N}$, using pure $\mathrm{AlN}$ as the barrier material leads to a stronger macroscopic polarization (both spontaneous and piezoelectric) and larger built-in electric fields. ${ }^{2,13}$ This offers a wider range of effective band-gap (optical emission) tunability of both interband transitions ${ }^{14}$ and improved intersubband transitions. ${ }^{15-17}$ In particular, earlier experimental results for using $(\mathrm{Ga}, \mathrm{Al}) \mathrm{N}$ as a barrier have shown that the band gap is set primarily by the thickness of the well layer, ${ }^{16}$ as well as by the barrier width. ${ }^{5}$ However, a systematic investigation, particularly from the theoretical side, of the dependence of the band gap of GaN/AlN (0001) multiple quantum wells (MQWs) (with a few dozen periods), on the thickness of both well and barrier is still missing. Regarding the strength of the electric field, a key parameter governing the optical properties of GaN/AlN heterostructures, and often used as an input parameter for nonselfconsistent simulations, ${ }^{4,18}$ most of the reported values show a wide disparity. Tight-binding simulations reported that the spontaneous and piezoelectric components are comparable, i.e., $1.14 \mathrm{MV} / \mathrm{cm}$ and $1.12 \mathrm{MV} / \mathrm{cm}$, respectively, for a
$\mathrm{GaN} / \mathrm{Al}_{0.2} \mathrm{Ga}_{0.8} \mathrm{~N}$ superlattice. ${ }^{3,4}$ However, Park and Chuang reported that for $\mathrm{GaN} / \mathrm{Al}_{0.2} \mathrm{Ga}_{0.8} \mathrm{~N} \mathrm{QW}$ structures, there exist strong spontaneous polarizations (about $1.1 \mathrm{MV} / \mathrm{cm}$ ) even when the piezoelectric field in the well is zero. ${ }^{5}$ By fitting the emission energy to the well width, the electric field was deduced to be $10 \pm 1 \mathrm{MV} / \mathrm{cm}$ (Ref. 7) in GaN/AlN (0001) MQWs, and 8-10 MV/cm (Refs. 14 and 19) in isolated QWs. The highest value of the electric field reported, by considering piezoelectric polarization alone, is $4.7 \mathrm{MV} / \mathrm{cm} .^{20}$ On the other hand, recent experiments reported that for well widths of 2.3 and $1.4 \mathrm{~nm}$ (and barrier width $1.9 \mathrm{~nm}$ ) the intrinsic electric field strengths in the wells were 5.04 $\mathrm{MV} / \mathrm{cm}$ and $6.07 \mathrm{MV} / \mathrm{cm},{ }^{16}$ respectively.

In this paper, we report a systematic first-principles investigation of the dependence of the band gap and built-in electric field as a function of the thickness of both well and barrier in GaN/AlN(0001) superlattices. The effect of strain on these two quantities is also studied. We find that the energy band gap and the built-in electric field of GaN/AIN (0001) superlattices can be tuned continuously by varying the thickness of both well and barrier. The calculated electric field strength values are in good agreement with recent experiments. Investigation into the effect of strain reveals that piezoelectric polarization only contributes about 5-10\% of the total macroscopic polarization.

\section{COMPUTATIONAL DETAILS}

MQWs, which consist of a large number of alternating well $(\mathrm{GaN})$ and barrier (AlN) layers, are simulated using periodic superlattices. We perform all-electron densityfunctional theory (DFT) calculations using the generalized gradient approximation (GGA) (Ref. 21) for the exchangecorrelation functional as implemented in the DMOL3 code. ${ }^{22}$ The wave functions are expanded in terms of a doublenumerical quality localized basis set with a real-space cutoff of 9 bohr. We construct various sized $(\mathrm{GaN})_{m} /(\mathrm{AlN})_{n}(0001)$ superlattices, where $m$ and $n$ are the number of double layers 


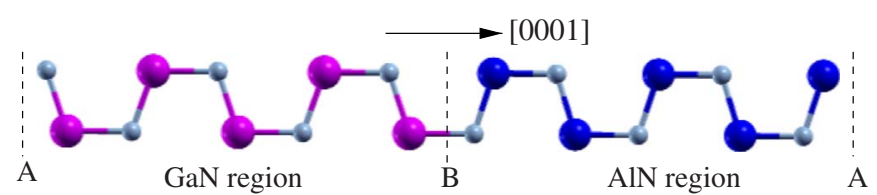

FIG. 1. (Color online) Geometry of the $(\mathrm{GaN})_{5} /(\mathrm{AlN})_{5}(0001)$ heterostructure. The center (middle dashed line) represents the type B interface while the two edges correspond to the type A interface. Small (green) spheres indicate $\mathrm{N}$ atoms and large spheres, light (pink) and dark (blue), indicate $\mathrm{Ga}$ and $\mathrm{Al}$ atoms, respectively.

of $\mathrm{GaN}$ and $\mathrm{AlN}$ in the well and barrier region, respectively. For short, we label it the " $m+n$ " superlattice. As an example, Fig. 1 shows the $5+5$ superlattice. It involves two different interfaces, "type A" and "type B." The Brillouin-zone integrations are performed using a $10 \times 10 \times 4 \mathbf{k}$-point grid for the $6+6$ structure. For other superlattices, the same/similar sampling of reciprocal space is used. We impose the assumption of pseudomorphic growth, i.e., the condition that the in-plane lattice constants of $\mathrm{GaN}$ region and AlN region are equal. Three approaches regarding the geometry relaxation are employed; (i) full relaxation, including lattice constants $(a$ and $c)$ and internal parameters, representing "freestanding" (strain-free) superlattices; then, in order to investigate the effect of strain, we fix the in-plane lattice constant of the superlattices at that of either (ii) GaN or (iii) AlN, relaxing the $c$ lattice constant and internal parameters.

As expected, DFT-GGA leads to an underestimation of the band-gap values for semiconductors. For example, our calculated direct band-gap values are $4.21 \mathrm{eV}$ and $1.81 \mathrm{eV}$, compared to the corresponding experimental values $6.12 \mathrm{eV}$ (Ref. 23) and $3.51 \mathrm{eV},{ }^{24}$ for AlN and GaN, respectively. To obtain a more accurate description of the band gap, and to compare the trend with varying well and barrier widths, we also perform self-interaction corrected local-density approximation (SIC-LDA) pseudopotential calculations ${ }^{25,26}$ for selected superlattices using the ABINIT code. ${ }^{27}$ The SIC-LDA calculated band-gap values are $6.96 \mathrm{eV}$ and $3.93 \mathrm{eV}$ for AlN and $\mathrm{GaN}$, respectively. Self-interaction corrections were included for the Ga $3 d$ and the $\mathrm{N} 2 s$ and $\mathrm{N} 2 p$ states in the nonlocal part of the respective pseudopotentials. ${ }^{28}$ In the SIC-LDA band-structure calculations we used the same relaxed structures as obtained in our DFT-GGA calculations.

\section{RESULTS AND DISCUSSIONS}

We start with $n=m$ superlattices. The calculated effective band-gap values, for layer thickness varying from 1 to 20 double layers (DLs) with a 1 DL increment, obtained using DFT-GGA, as well as several selected values by SIC-LDA, are displayed in Fig. 2(a). Both methods predict that the band gap decreases monotonically with increasing well and barrier width. In the interband transition regime, laser diodes generate light via the process of electron-hole recombination, and the energy of the photon and hence the emission wavelength is therefore determined by the band gap. Our results thus show that the emission energy can be tuned continuously by changing the quantum well and barrier thickness over a large range. The DFT-GGA calculations predict that metallization
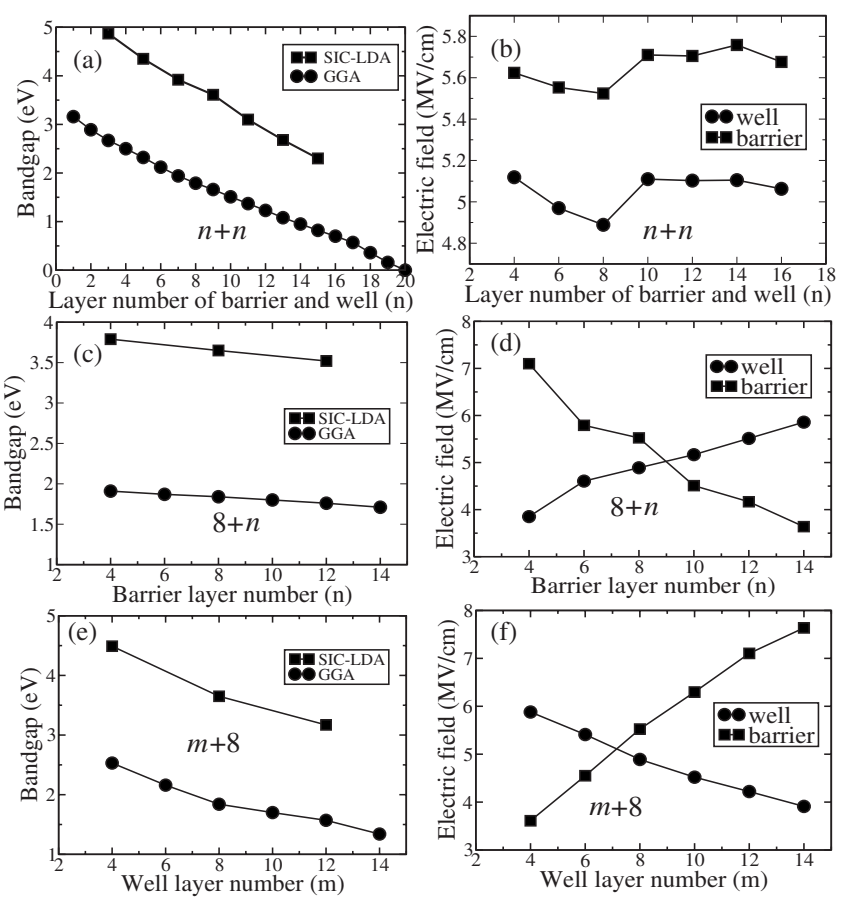

FIG. 2. Calculated band-gap values (left) and the absolute values for the strength of the built-in electric field (right) for various free-standing superlattices.

occurs at around $n=m=20$. As expected, the SIC-LDA bandgap values are systematically larger than those obtained from GGA by around $2 \mathrm{eV}$, and the predicted (by linearly extrapolating) metallization occurs at around $n=m=28$. The bandgap evolution as a function of thickness changes from the quantum confinement (blueshift) regime to the QCSE (redshift) regime. The bulk well (GaN) band-gap values [GGA: $1.82 \mathrm{eV}$, SIC-LDA: $4.01 \mathrm{eV}$, and expt.: $3.475 \mathrm{eV}$ (Ref. 16)] separate these two regions, which for both DFT-GGA and SIC-LDA results occurs around the $7+7$ superlattice. Note in the small-thickness region, the electric field induced redshift concedes with the strong confinement blueshift, which accounts for the more dramatic increase in band gap against decreasing thickness.

The presence of a built-in electric field can be demonstrated by using the core levels as reference energies to determine the relative alignment of the valence-band edges..$^{29,30}$ Here we use the N $1 s$ orbital binding energy, $E_{b}^{\mathrm{CL}}$, in different layers, as shown in Fig. 3(a) for the various superlattices. There is a different slope of the curves in the well GaN (positive) and barrier AlN (negative) regions, showing V-shaped profiles and the electric fields have opposite signs at the two sides of the interface. The slopes of the linear regions correspond to the absolute values of the electric fields $^{30}\left(|E|=\Delta E_{b}^{\mathrm{CL}} / \Delta z\right)$, where $\Delta E_{b}^{\mathrm{CL}}$ is the difference, $E_{b}^{\mathrm{CL}}$, of the $\mathrm{N}$ atoms with a distance of $\Delta z$ along the [0001] direction. The calculated absolute values in the well and barrier regions are about $5 \pm 0.1 \mathrm{MV} / \mathrm{cm}$ and $5.6 \pm 0.1 \mathrm{MV} / \mathrm{cm}$, respectively, for $n=m$ superlattices [Fig. 2(b)]. The periodic condition of superlattices imply that $E_{b} / E_{w}=-L_{w} / L_{b},{ }^{5}$ where superscripts " $w$ " and " $b$ " represent the well and barrier, and $E$ and $L$ are the strength of electric field and layer thickness, 

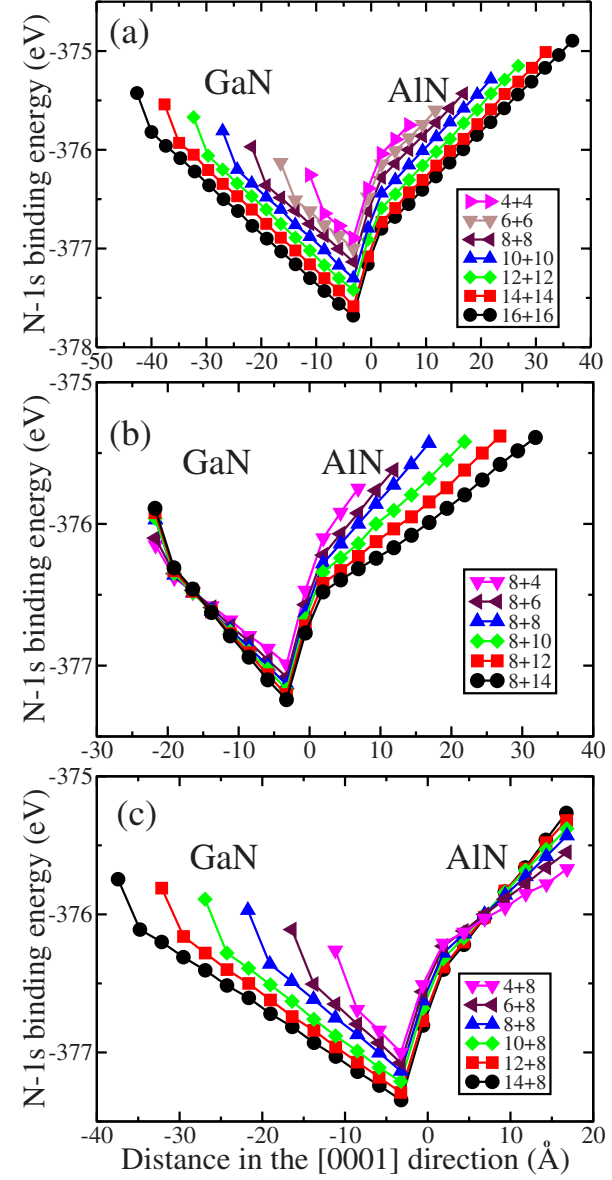

FIG. 3. (Color online) Calculated variation in the $\mathrm{N} 1 s$ corelevel binding energy (electron volt) along the [0001] direction for (a) $n+n$; (b) $n+8$, and (c) $8+n$ free-standing superlattices.

respectively. For the cases of $n=m$, the larger magnitude in the barrier region is due to the slightly shorter AlN layer $(2.49 \AA)$ than the GaN layer $(2.64 \AA)$.

Such strong electric fields correspond to a dramatic charge accumulation and large monopole around the interfaces, ${ }^{12,31}$ and have significant effects on the electronic structure of the superlattices. The total band structure and layer-resolved [each layer containing one metal $(\mathrm{Ga}$ or $\mathrm{Al})$ and one $\mathrm{N}$ atom] density of states (DOS) are demonstrated, as an example, in Fig. 4 for the $16+16$ superlattice. First, the existence of the electric field leads to a bending of the conduction- and valence-band profiles across the superlattice
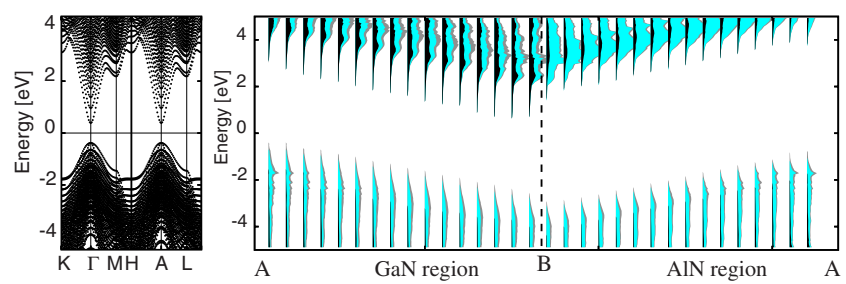

FIG. 4. (Color online) Total band-structure (left) and layerresolved DOS (right) for the free-standing 16+16 superlattice. Dark (black) represents the $s$ orbital, light (green) the $p$ orbital, and gray the $d$ orbital. The dashed line indicates the type B interface. and the related localization of electron and hole gases on the two opposite interfaces of the quantum well. The resulted valence-band maximum (VBM) around interface $\mathrm{A}$ and the conduction-band minimum (CBM) at interface B determine the overall band gap of superlattices. With increasing well and barrier thickness, the strong built-in electric field in the AlN/GaN superlattices gives rise to a marked reduction in the effective band gap. Second, the progressive separation of the VBM and CBM with increasing thickness, particularly the well thickness, will diminish the electron-hole recombination process (emission strength), and thus the matrix element of the optical transition due to the decrease in the electron and hole wave functions overlap. ${ }^{4}$ The latter adverse effect has lead to the conclusion that for optimal optical devices (such as QW lasers), the maximum well thickness should be around $3 \mathrm{~nm}(12 \mathrm{DL} \mathrm{GaN}) \cdot{ }^{13,14}$ Note that in Ref. 14, the large linewidth broadening indicated severe thickness fluctuations and interface roughness, which may contribute to a prevalence of nonradiative recombination. A large oscillator strength was achieved for a $4 \mathrm{~nm}$ well thickness in $\mathrm{GaN} / \mathrm{Al}_{0.15} \mathrm{Ga}_{0.85} \mathrm{~N}$ samples ${ }^{4}$ and up to $8 \mathrm{~nm}$ in $\mathrm{GaN} / \mathrm{Al}_{0.17} \mathrm{Ga}_{0.83} \mathrm{~N} .^{2}$

The feasibility of tuning the band gap of MQW structures is further boosted by changing only either well or barrier thickness while keeping the thickness of the other one unchanged. To demonstrate this, we report a well-thickness and barrier-thickness study by using $m+8(m=4,6,8,10,12$, and 14) and $8+n(n=4,6,8,10,12$, and 14) superlattices. The calculated band-gap values and the strength of the electric field in the well and barrier regions are shown in Fig. 2, and the plots of core level $\mathrm{N} 1 s$ binding energies are shown in Fig. 3.

Increasing either well or barrier thickness will lead to a decrease in the band gap, as is predicted by both the GGA and SIC-LDA calculations. As expected, changing the well thickness is more effective because as a type I heterostructure system, i.e., the VBM (CBM) of GaN is higher (lower) than that of AlN, the band gap of the GaN/AlN(0001) superlattice is mainly determined by the well $\mathrm{GaN}$ region. Increasing the thickness of either the barrier or well will lead to a decrease in the magnitude of the electric field in the same region and an increase in the other region. More importantly, compared to the modest change in the electric field for $n=m$ superlattices, such a change for the $n \neq m$ cases is much more efficient. Thus, our results demonstrate that the values of the band gap and the built-in electric field exhibit a significant dependence on both the well and the barrier widths, which offers the possibility to tune the emission energy over a wide range. In addition, by varying the thickness of both well and barrier, one can realize multitarget band-gap design. ${ }^{32}$ For example, $9+9,12+8$, and $8+14$ superlattices give a target band gap of $1.65 \mathrm{eV}$ (DFT-GGA).

So far, we have only considered free-standing, i.e., strainfree superlattices. Biaxial strain perpendicular to the [0001] direction will induce a piezoelectric field along the [0001] direction. Fixing the lateral lattice constant of the superlattices at that of GaN (fix-GaN), and of AlN (fix-AlN), represents two "extremes" of the compressive and tensile-strained superlattices. For this purpose, we consider $6+8,8+8$, and 
TABLE I. Calculated lattice constant $c$ in the [0001] direction (angstrom) and the built-in electric field in the well (GaN) region, $E_{w}$, and in the barrier $(\mathrm{AlN})$ region, $E_{b}$, (megavolt per centimeter).

\begin{tabular}{|c|c|c|c|c|c|c|c|c|c|}
\hline \multirow[b]{2}{*}{ Strain type } & \multicolumn{3}{|c|}{$\begin{array}{c}c \\
(\AA)\end{array}$} & \multicolumn{3}{|c|}{$\begin{array}{c}E_{w} \\
(\mathrm{MV} / \mathrm{cm})\end{array}$} & \multicolumn{3}{|c|}{$\begin{array}{c}E_{b} \\
(\mathrm{MV} / \mathrm{cm})\end{array}$} \\
\hline & $6+8$ & $8+8$ & $10+8$ & $6+8$ & $8+8$ & $10+8$ & $6+8$ & $8+8$ & $10+8$ \\
\hline Fix AlN & 36.07 & 41.41 & 46.76 & 5.19 & 4.72 & 4.24 & 4.36 & 4.77 & 6.01 \\
\hline Fix GaN & 35.47 & 40.71 & 45.94 & 6.09 & 5.19 & 4.76 & 5.08 & 5.19 & 6.81 \\
\hline Free standing & 35.74 & 40.97 & 46.20 & 5.41 & 4.89 & 4.52 & 4.55 & 4.89 & 6.30 \\
\hline
\end{tabular}

$10+8$ superlattices. The calculated in-plane lattice parameters of pure AlN (3.134 $\AA$ ) and GaN (3.228 $\AA$ ) differ by $2.91 \%$, compared to the experimental value of $2.41 \% .{ }^{24}$ The calculated in-plane lattice constant for the free-standing 6 $+8,8+8$, and $10+8$ superlattices are $3.162 \AA, 3.168 \AA$, and $3.172 \AA$, respectively. For these superlattices, the calculated $c$ lattice constants and strength of the electric fields are listed in Table I, and the band-gap values in Table II.

In the GaN/AlN(0001) heterostructure, the direction of spontaneous polarization is from the nitrogen (anion) atom to the nearest-neighbor metal (cation) atom along the $c$ axis, i.e., the $[000 \overline{1}]$ direction. ${ }^{1,33}$ The alignment of the piezoelectric and spontaneous polarization is parallel in the case of tensile strain and antiparallel for compressive strain. ${ }^{33}$ This is in agreement with our calculated values shown in Table I, where the calculated strength of the electric field values in the well and barrier regions for fix-AlN (fix-GaN) are smaller (larger) than the corresponding free-standing ones. Our calculated values are in good agreement with recent experimental results, ${ }^{16}$ namely, 6.07 and $5.04 \mathrm{MV} / \mathrm{cm}$ for the 20 period $2.3 \mathrm{~nm} / 1.9 \mathrm{~nm}$ and $1.4 \mathrm{~nm} / 1.9 \mathrm{~nm}$ [corresponding to $(8-10)+8$ and $(5-6)+8]$ GaN/AlN MQWs. Moreover, in all the superlattices considered, the magnitudes of the electric fields in the well region are around 4-6 MV/cm, which are significantly lower than the $10 \pm 1 \mathrm{MV} / \mathrm{cm}$ (Ref. 7) deduced by fitting spectra. It is known that dielectric screening and geometrical factors also affect the polarization fields in III-V nitride heterostructures. ${ }^{2,3}$ Consequently, the electric field of single QWs is expected to be larger than that in MQWs, as obtained in Refs. 14 and 19. Note, our study shows that the field strength is a function of supercell thickness, not a constant as assumed in Ref. 14. Moreover, we confirm that the significant built-in electric field is dominantly due to spontaneous polarization. Comparison of the electric field for the three strain conditions reveals that piezoelectric polarization plays only a minor role, estimated to be $5-10 \%$.
Another approach to estimate the electric filed is based on $|E|=\Delta E_{\text {gap }} / \Delta d$, where $\Delta E_{\text {gap }}$ is the difference of the band gap with the changes of the superlattice thickness, $\Delta d{ }^{3}$ Assuming the band edge changes linearly with the field, this method gives an averaged electric field of $3.2 \mathrm{MV} / \mathrm{cm}$ by DFT-GGA and 3.9 MV/cm by LDA-SIC for the $n+n$ superlattices in Fig. 2(a). The underestimated values are partial due to the fact that such a method neglects the contribution of the quantum confinement, which also plays a significant role in affecting the band-gap values, particularly in short superlattices. Indeed, for shorter than $7+7$ superlattices, the estimated electric field is about $4.6 \mathrm{MV} / \mathrm{cm}$ by DFT-GGA.

Strain effects also influence the band gap, both the DFTGGA and SIC-LDA calculations demonstrate that compressive (tensile) strain leads to larger (smaller) band-gap values compared to the free-standing superlattices with the exception of $6+8$ by SIC-LDA. Such a small deviation may be related to the fact that we have used the relaxed DFT-GGA structures for the SIC-LDA calculations. Thus, strain offers another degree of freedom for tuning the band gap and electric field.

\section{CONCLUSION}

To summarize, our first-principles DFT calculations systematically demonstrate the continuous tunability of the band gap and the strength of the built-in electric fields in GaN/ AlN (0001) superlattices by adjustment of the thickness of both the well $(\mathrm{GaN})$ and barrier (AlN) regions leaving much room for future improvement and tailoring of various nitride devices. The effects of strain in affecting the band gap and electric field are also established. We confirm that spontaneous polarization plays a dominant role for the large built-in electric fields, typically 4-6 MV/cm, while strain-induced piezoelectric polarizations contribute only about $5-10 \%$.

TABLE II. Calculated band-gap values for strained and free-standing GaN/AlN superlattices from GGA and SIC-LDA. Units are in electron volt.

\begin{tabular}{lcccccc}
\hline \hline & \multicolumn{3}{c}{ GGA } & \multicolumn{3}{c}{ SIC-LDA } \\
\hline Strain type & $6+8$ & $8+8$ & $10+8$ & $6+8$ & $8+8$ & $10+8$ \\
Fix AlN & 2.13 & 1.84 & 1.67 & 4.12 & 3.78 & 3.57 \\
Fix GaN & 2.00 & 1.75 & 1.56 & 3.98 & 3.63 & 3.35 \\
Free standing & 2.08 & 1.80 & 1.60 & 4.21 & 3.76 & 3.49 \\
\hline \hline
\end{tabular}




\section{ACKNOWLEDGMENTS}

We acknowledge the computing resources provided by the NCI National Facility in Canberra, Australia, which is supported by the Australian Commonwealth Government, and from the NSF (U.S.) (through its MRSEC program at the Northwestern Materials Research Center).
${ }^{1}$ F. Bernardini, V. Fiorentini, and D. Vanderbilt, Phys. Rev. B 56, R10024 (1997).

${ }^{2}$ N. Grandjean, B. Damilano, S. Dalmasso, M. Leroux, M. Laugt, and J. Massies, J. Appl. Phys. 86, 3714 (1999).

${ }^{3}$ V. Fiorentini, F. Bernardini, F. Della Sala, A. Di Carlo, and P. Lugli, Phys. Rev. B 60, 8849 (1999).

${ }^{4}$ R. Cingolani, A. Botchkarev, H. Tang, H. Morkoc, G. Traetta, G. Coli, M. Lomascolo, A. Di Carlo, F. Della Sala, and P. Lugli, Phys. Rev. B 61, 2711 (2000).

${ }^{5}$ S.-H. Park and S.-L. Chuang, Appl. Phys. Lett. 76, 1981 (2000).

${ }^{6}$ K. Omae, Y. Kawakami, S. Fujita, M. Yamada, Y. Narukawa, and T. Mukai, Phys. Rev. B 65, 073308 (2002).

${ }^{7}$ M. Tchernycheva, L. Nevou, L. Doyennette, F. H. Julien, E. Warde, F. Guillot, E. Monroy, E. Bellet-Amalric, T. Remmele, and M. Albrecht, Phys. Rev. B 73, 125347 (2006).

${ }^{8}$ E. P. Pokatilov, D. L. Nika, and A. A. Balandin, Appl. Phys. Lett. 89, 113508 (2006).

${ }^{9}$ M. Leroux, N. Grandjean, M. Laugt, J. Massies, B. Gil, P. Lefebvre, and P. Bigenwald, Phys. Rev. B 58, R13371 (1998).

${ }^{10}$ J. S. Im, H. Kollmer, J. Off, A. Sohmer, F. Scholz, and A. Hangleiter, Phys. Rev. B 57, R9435 (1998).

${ }^{11}$ J. A. Majewski, S. Hachenbuchner, G. Zandler, and P. Vogl, Comput. Mater. Sci. 30, 81 (2004).

${ }^{12}$ X. Y. Cui, J. E. Medvedeva, B. Delley, A. J. Freeman, and C. Stampfl, Phys. Rev. B 78, 245317 (2008).

${ }^{13}$ N. Grandjean, J. Massies, and M. Leroux, Appl. Phys. Lett. 74, 2361 (1999).

${ }^{14}$ C. Adelmann, E. Sarigiannidou, D. Jalabert, Y. Hori, J.-L. Rouviere, B. Daudin, S. Fanget, C. Bru-Chevallier, T. Shibata, and M. Tanaka, Appl. Phys. Lett. 82, 4154 (2003).

${ }^{15}$ E. Baumann, F. R. Giorgetta, D. Hofstetter, S. Golka, W. Schrenk, G. Strasser, L. Kirste, S. Nicolay, E. Feltin, J. F. Carlin, and N. Grandjean, Appl. Phys. Lett. 89, 041106 (2006).

${ }^{16}$ C. Buchheim, R. Goldhahn, A. T. Winzer, G. Gobsch, U. Rossow, D. Fuhrmann, A. Hangleiter, F. Furtmayr, and M. Eickhoff, Appl. Phys. Lett. 90, 241906 (2007).

${ }^{17}$ C. Bayram, N. Pere-laperne, R. McClintock, B. Fain, and M. Razeghi, Appl. Phys. Lett. 94, 121902 (2009).

${ }^{18}$ N. Iizuka, K. Kaneko, and N. Suzuki, Appl. Phys. Lett. 81, 1803 (2002).

${ }^{19}$ A. Helman, M. Tchernycheva, A. Lusson, E. Warde, F. H. Julien, Kh. Moumanis, G. Fishman, E. Monroy, B. Daudin, D. Le Si Dang, E. Bellet-Amalric, and D. Jalabert, Appl. Phys. Lett. 83,
5196 (2003).

${ }^{20}$ G. Martin, A. Botchkarev, A. Rockett, and H. Morkoc, Appl. Phys. Lett. 68, 2541 (1996).

${ }^{21}$ J. P. Perdew, K. Burke, and M. Ernzerhof, Phys. Rev. Lett. 77, 3865 (1996).

${ }^{22}$ B. Delley, J. Chem. Phys. 113, 7756 (2000); 92, 508 (1990).

${ }^{23}$ J. Li, K. B. Nam, M. L. Nakarmi, J. Y. Lin, H. X. Jiang, P. Carrier, and S.-H. Wei, Appl. Phys. Lett. 83, 5163 (2003).

${ }^{24}$ I. Vurgaftman and J. R. Meyer, J. Appl. Phys. 94, 3675 (2003).

${ }^{25}$ D. Vogel, P. Kruger, and J. Pollmann, Phys. Rev. B 55, 12836 (1997); Specifically, we use the Troullier-Martins scheme [N. Troullier and J. L. Martins, ibid. 43, 1993 (1991)]; as implemented in the FHIPP code [M. Fuchs and M. Scheffler, Comput. Phys. Commun. 119, 67 (1999)] to generate the SIC pseudopotentials.

${ }^{26}$ A. Qteish, A. I. Al-Sharif, M. Fuchs, M. Scheffler, S. Boeck, and J. Neugebauer, Phys. Rev. B 72, 155317 (2005).

${ }^{27}$ X. Gonze, J.-M. Beuken, R. Caracas, F. Detraux, M. Fuchs, G.-M. Rignanese, L. Sindic, M. Verstraete, G. Zerah, F. Jollet, M. Torrent, A. Roy, M. Mikami, P. Ghosez, J.-Y. Raty, and D. C. Allan, Comput. Mater. Sci. 25, 478 (2002).

${ }^{28}$ Troullier-Martins pseudopotentials were constructed in atomic LDA calculations for $\mathrm{Al}\left(3 s^{2}, 3 p^{3}, 3 d^{0}\right), \mathrm{Ga}\left(3 d^{10}, 4 s^{2}, 4 p^{1}\right)$, and $\mathrm{N}\left(2 s^{2}, 2 p^{3}, 3 d^{0}\right)$ with pseudoization radii $\left(r_{s}, r_{p}, r_{d}\right)$ of $(1.5,1.5$, $1.5)$ bohr, $(2.2,2.4,2.1)$ bohr, and $(1.8,2.0,2.1)$ bohr, respectively. SIC potentials were then added for the Ga $3 d$ and $\mathrm{N} 2 s$ and $\mathrm{N} 2 p$ states, constructed with a cutoff radius of $7.3 \mathrm{bohr}$. The potentials were used in Kleinman-Bylander form [L. Kleinman and D. M. Bylander. Phys. Rev. Lett. 48, 1425 (1982).] with the $d$-( $\mathrm{Al}$ and $\mathrm{N})$ and $s$-components $(\mathrm{Ga})$ as local potentials and checked to be free of ghost states.

${ }^{29}$ S. Massidda, B. I. Min, and A. J. Freeman, Phys. Rev. B 35, 9871 (1987).

${ }^{30}$ S. Picozzi, A. Continenza, and A. J. Freeman, Phys. Rev. B 55, 13080 (1997).

${ }^{31}$ F. Bernardini and V. Fiorentini, Phys. Rev. B 57, R9427 (1998).

${ }^{32}$ P. Piquini, P. A. Graf, and A. Zunger, Phys. Rev. Lett. 100, 186403 (2008).

${ }^{33}$ O. Ambacher, J. Smart, J. R. Shealy, N. G. Weimann, K. Chu, M. Murphy, W. J. Schaff, L. F. Eastman, R. Dimitrov, L. Wittmer, M. Stutzmann, W. Rieger, and J. Hilsenbeck, J. Appl. Phys. 85, 3222 (1999). 